Check for updates

Cite this: RSC Adv., 2017, 7, 56280

\title{
The role of semaphorin 7A and its receptor plexin C1 in the migration of NSCLC cells
}

\author{
Minlong Zhang, $\mathbb{D} \dagger$ Hu Wang† and Faguang Jin* \\ We aim to explore the role of semaphorin 7A (SEMA7A) and its receptor plexin C1 in the migration of NSCLC \\ cells. In the present study, expression of SEMA7A and plexin C1 in A549 cells and H1299 cells were detected by \\ western blot. The effect of interaction between SEMA7A and plexin C1 in cell migration and migration related \\ signaling pathway was detected using recombinant SEMA7A and plexin C1 small interfering RNA (siRNA). Both \\ cells showed an increase in SEMA7A and plexin C1 expression compared with normal alveolar epithelial cells. In \\ addition, SEMA7A activated tumor migration by increasing phosphorylation of its related protein FAK, LIMKII \\ and actin-binding protein cofilin, which are also downstream targets of plexin C1. However, SEMA7A failed \\ to activate cell migration and its related protein FAK, LIMKII and cofilin when plexin C1 was silenced. These \\ results suggest that the interaction between SEMA7A and plexin C1 promoted migration of A549 cells and \\ H1299 cells and this effect worked through actin-binding protein cofilin signaling activation.
}

Received 2nd August 2017

Accepted 8th December 2017

DOI: 10.1039/c7ra08518d

rsc.li/rsc-advances

SEMA7A can also skew macrophages into a pro-tumorigenic state to promote tumor progression in mammary carcinoma. ${ }^{\mathbf{1 2}}$ However, interaction between SEMA7A and plexin C1 plays a different role in melanoma. ${ }^{13}$ Plexin $\mathrm{C} 1$ is absent in human melanoma cell lines and the loss of plexin $\mathrm{C} 1$ has been regarded as a critical factor of melanoma invasion and metastasis.

Focal adhesion kinase (FAK) and cofilin pathway are strongly linked to the tumor adhesion, migration and progression. ${ }^{\mathbf{1 4 - 1 9}}$ Cofilin is an actin binding protein involved in actin disassembly and is downstream target of LIM kinases (LIMK), which can phosphorylate cofilin on Ser-33. LIMK include two isoforms, LIMKI and LIMKII. ${ }^{20}$ LIMKI is only expressed in neural cells and LIMKII is widely expressed. Previous study has showed that cofilin is also a downstream target of plexin $\mathrm{C} 1 .{ }^{13}$ Recently, we found that SEMA7A and plexin C1 were highly expressed in lung cancer cells. However, the role of SEMA7A-plexin C1 in NSCLC (non-small cell lung cancer) cell migration and its specific mechanism are not clear.

In this study, we explored the interaction between SEMA7A and its receptor plexin C1 in lung cancer A549 cell and H1299 cell migration. In addition, we also investigated the changes of tumor migration related signaling pathway. The results of our study suggested that SEMA7A and plexin C1 highly expressed in both cells. The interaction between SEMA7A and its receptor plexin $\mathrm{C} 1$ promoted the migration of both cells and activated tumor migration related proteins FAK, LIMKII and cofilin.

\section{Materials and methods}

Department of Respiration, Tangdu Hospital, Fourth Military Medical University, Xi'an 710038, PR China. E-mail: jinfag@fmmu.edu.cn; Fax: +86 29 84777425; Tel: $+862984777425$

$\dagger$ These authors contributed to the article equally.

\subsection{Cell culture and siRNA transfection}

Human pulmonary alveolar epithelial cells (obtained from Sciencell, San Diego, CA, USA) and human non-small cell lung 
cancer (NSCLC) cell line A549 and H1299 (obtained from ATCC, Rockville, MD, USA), were maintained in RPMI 1640 medium supplemented with $100 \mathrm{U} \mathrm{ml}^{-1}$ of penicillin-streptomycin and $10 \%$ fetal bovine serum (FBS) at $37{ }^{\circ} \mathrm{C}$ in a humidified atmosphere containing $5 \% \mathrm{CO}_{2}$ and $95 \%$ air. Specific siRNA for plexin $\mathrm{C} 1$ and the scramble control siRNA were purchased from Genechem (Shanghai, China). The target sequence used against human plexin C1 was as follows: $5^{\prime}$-CCCTTCCTTGACTACAAA CAT-3'. The siRNA was transiently transfected into cells with Lipofectamine 2000 (Invitrogen) according to manufacturer's instructions. $48 \mathrm{~h}$ after transfection, cells were treated with or without Recombinant SEMA7A (rSEMA7A) (obtained from R\&D Systems, Minneapolis, MN, USA).

\subsection{Determination of mRNA}

Total RNA was extracted with TRIZOL reagent (Takara, Dalian, China) according to the manufacturer's instruction. RNA concentration was tested by spectrometric analysis. SEMA7A, plexin C1, $\beta 1$-integrin and $\beta$-actin were examined by Real Time PCR following the manufacturer's instructions (Takara Perfect Real Time). Amplification and detection were carried out by using Bio-Rad My iQ detection system. Relative quantification

A
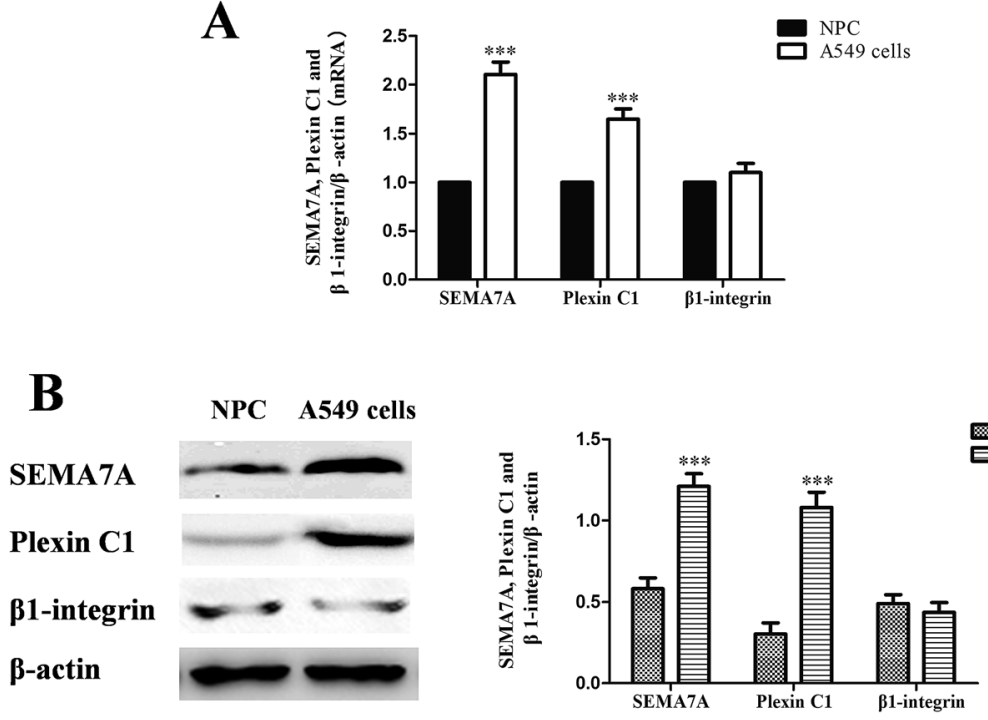

Fig. 1 SEMA7A and its receptor plexin C1 upregulated in NSCLC A549 cells. (NPC) normal human pulmonary alveolar epithelial cells group. (A) mRNA expression of SEMA7A, plexin C1 and $\beta 1$-integrin (real-time RT PCR). (B) Protein expression of SEMA7A, plexin C1 and $\beta 1$-integrin (western blot). $n=8, * * * P<0.001$ versus group NPC
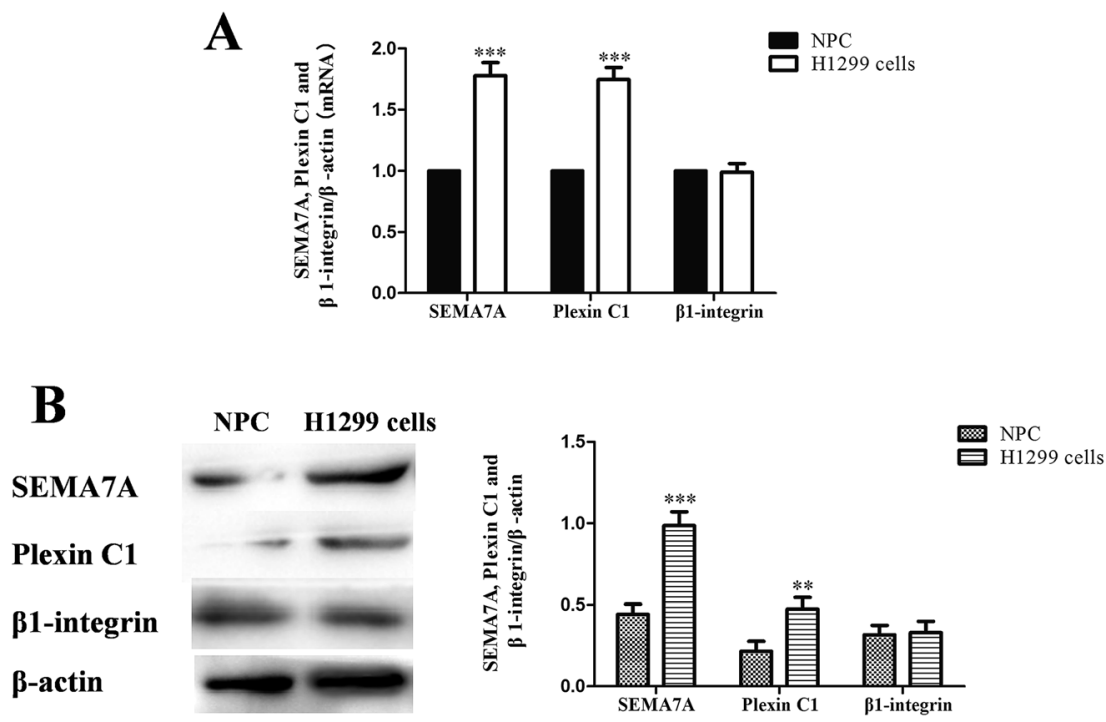

Fig. 2 SEMA7A and its receptor plexin C1 upregulated in NSCLC H1299 cells. (NPC) normal human pulmonary alveolar epithelial cells group. (A) mRNA expression of SEMA7A, plexin C1 and $\beta 1$-integrin (real-time RT PCR). (B) Protein expression of SEMA7A, plexin C1 and $\beta 1$-integrin (western blot). $n=8, * * P<0.01, * * * P<0.001$ versus group NPC. 
of target cDNA was determined by arbitrarily setting the control value to 1 and changes in cDNA content of a sample were expressed as a multiple thereof. Genes and primers are listed as follows: the sequences of the human SEMA7A primers were $5^{\prime}$ CACCATCCGGAAGCAGGAATA- $3^{\prime}$ (forward) and $5^{\prime}$-TGCAC GATGGTGGCTTTGA-3' (reverse). The sequences of the human plexin C1 primers were $5^{\prime}$-CTTGCTGCCGCCACCG-3' (forward) and $5^{\prime}$-TCGCCCAGGAAGGAGAGC-3' (reverse). The sequences of the human $\beta 1$-integrin primers were $5^{\prime}$-CCGCGGCTGGT TTCCTG- $3^{\prime}$ (forward) and 5'-CTGCTGTTCCGCGACTCC- $3^{\prime}$ (reverse).

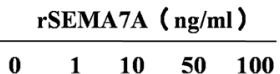

P-FAK

FAK

P-cofilin

cofilin

LIMK II

$\beta$-actin
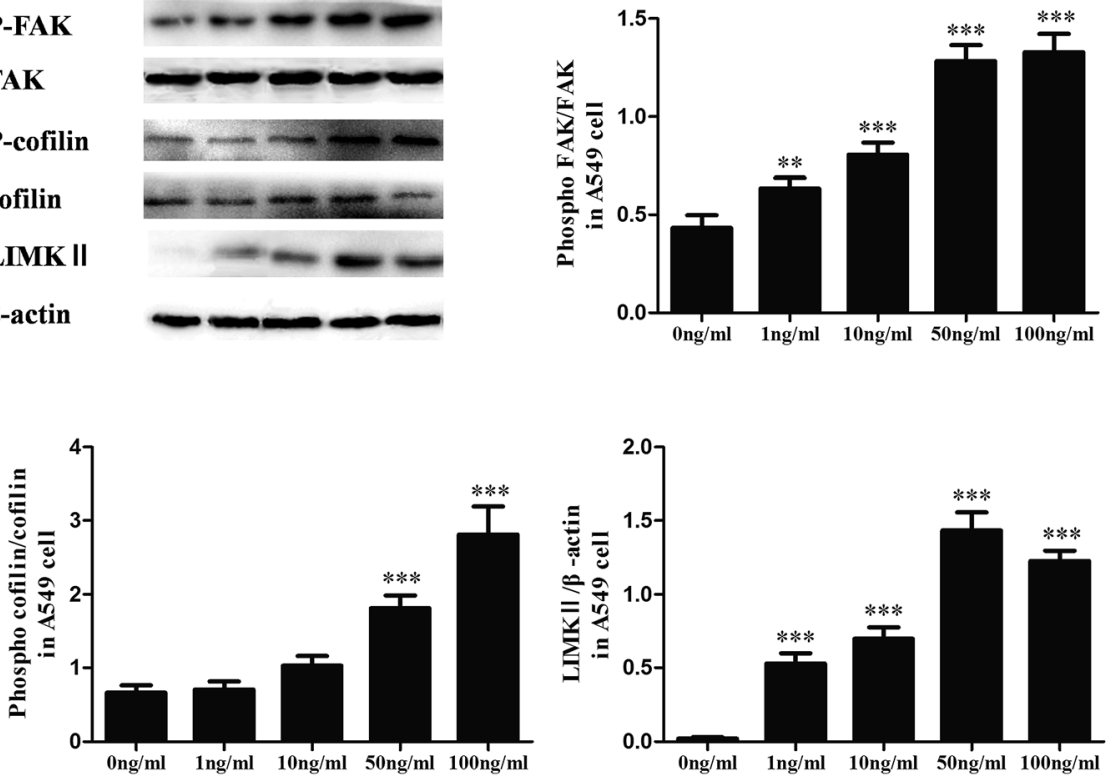

Fig. 3 SEMA7A activates the expression of LIMKII, stimulates phosphorylation of FAK and cofilin in NSCLC A549 cells. All data were obtained at 15 min after rSEMA7A stimulation. $n=8, * * P<0.01, * * * P<0.001$ versus $0 \mathrm{ng} \mathrm{ml}^{-1}$ rSEMA7A group.
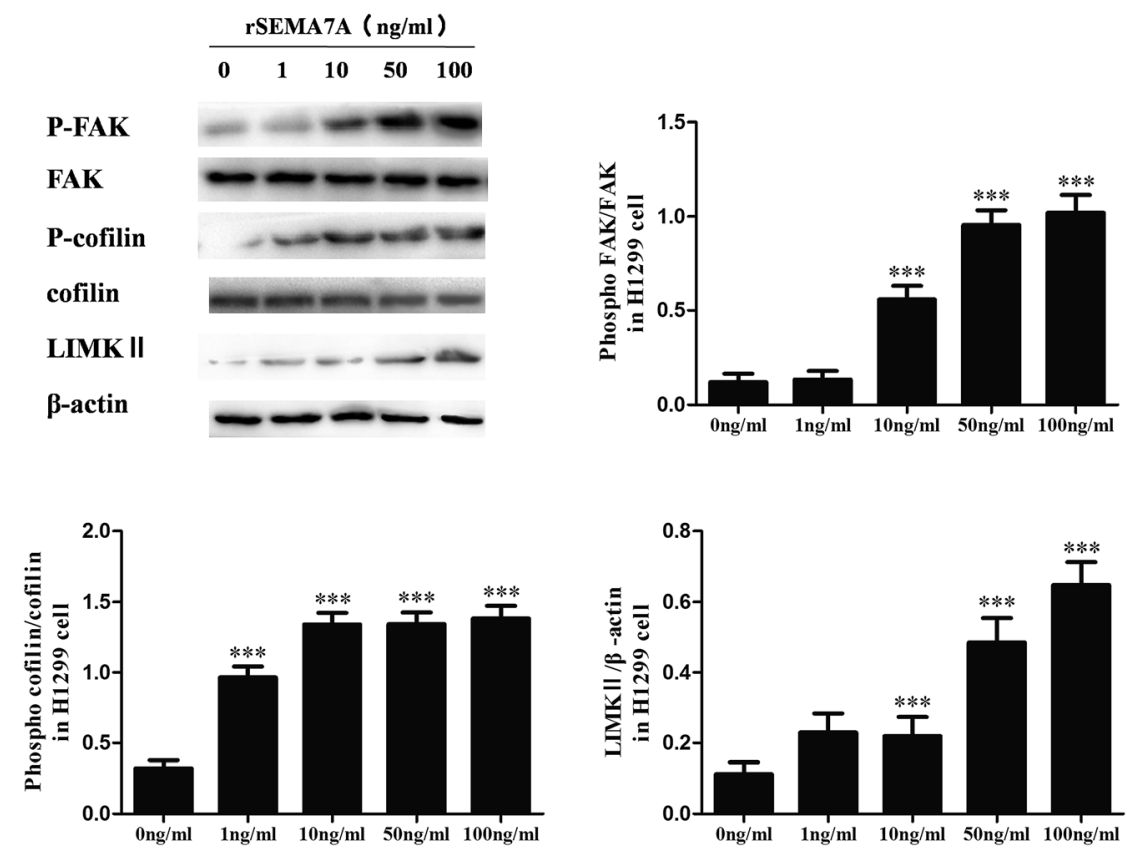

Fig. 4 SEMA7A activates the expression of LIMKII, stimulates phosphorylation of FAK and cofilin in NSCLC H1299 cells. All data were obtained at 15 min after rSEMA7A stimulation. $n=8, * * * P<0.001$ versus $0 \mathrm{ng} \mathrm{ml}^{-1}$ rSEMA7A group. 
The sequences of the human $\beta$-actin primers were $5^{\prime}$ TGGCACCCAGCACAATGAA-3' (forward) and 5'-CTAAGTCATA GTCCGCCTAGAAGCA-3' (reverse).

\subsection{Western blot analysis}

Cells proteins $\left(1 \times 10^{6}\right.$ cells per $\left.\mathrm{ml}\right)$ were extracted according to instructions of protein extraction kit and protein concentration was determined by BCA protein assay kit. Proteins (35 $\mu \mathrm{g}$ per sample) were separated in $10 \%$ SDS-polyacrylamide gels and electrotransferred to nitrocellulose (NC) membranes. The membrane was incubated overnight with rabbit monoclonal antibodies for $\beta$-actin (1:5000 dilution, $0.2 \mu \mathrm{g} \mathrm{ml} \mathrm{m}^{-1}$, Santa
Cruz, CA, USA), SEMA7A (1 : 1000 dilution, $1 \mu \mathrm{g} \mathrm{m} \mathrm{m}^{-1}$, Abcam, Cambridge, UK), plexin $\mathrm{C} 1$ and $\beta 1$-integrin (1: 1000 dilution, 1 $\mu \mathrm{g} \mathrm{ml} \mathrm{m}^{-1}$, R\&D Systems, Minneapolis, MN, USA), LIMKII (1 : 1000

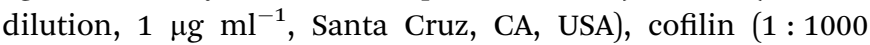
dilution, $1 \mu \mathrm{g} \mathrm{ml}^{-1}$, Cell Signaling, MA, USA), p-cofilin (1 : 1000 dilution, $1 \mu \mathrm{g} \mathrm{ml}^{-1}$, Cell Signaling, MA, USA). The secondary antibody $\left(1: 5000,2 \mu \mathrm{g} \mathrm{ml}^{-1}\right)$ was incubated. Detection of target protein uses enhanced chemiluminescent (ECL) detection system (Amersham, Arlington Heights, IL, USA). The membrane was fully covered with ECL, and scanned with the ChemiDoc MP Imaging System (Bio-Rad, Hercules, CA, USA). The integrated optical density (IOD) value of each band was calculated with
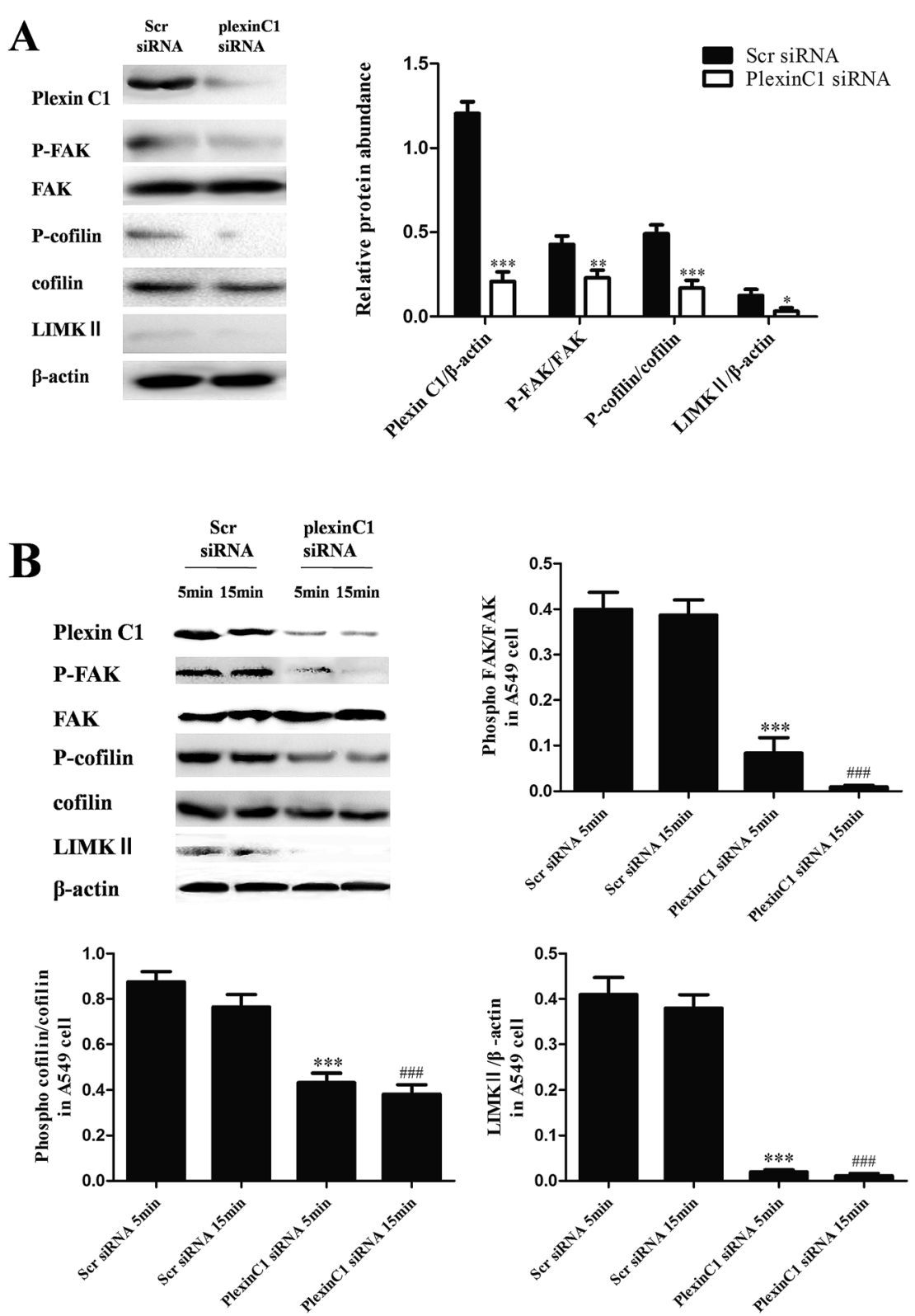

Fig. 5 Knockdown of plexin C1 decreases the expression of LIMKII, inhibits phosphorylation of FAK and cofilin in NSCLC A549 cells. (A) All data were obtained with $0 \mathrm{ng} \mathrm{m}^{-1}$ rSEMA7A stimulation. $n=8, * P<0.05, * * p<0.01$ and $* * * P<0.001$ versus group Scr siRNA. (B) All data were obtained with $100 \mathrm{ng} \mathrm{ml}^{-1}$ rSEMA7A stimulation for 5 or 15 min. $n=8, * * * P<0.001$ versus rSEMA7A for 5 min + scramble siRNA group, \#\#\#P< 0.001 versus rSEMA7A for 15 min + scramble siRNA group. 
Image lab software (Bio-Rad, Hercules, CA, USA), and the relative expressions of the target proteins were calculated by the ratio of the IOD value of the target band and the IOD value of the corresponding internal reference ( $\beta$-actin).

\subsection{Wound scratch assay}

A549 cells or H1299 cells $\left(2 \times 10^{5}\right.$ cells per hole in 6 wells plates $)$ were seeded in growth medium and the siRNA was transiently transfected into cells. $48 \mathrm{~h}$ after transfection, cells were treated with or without rSEMA7A. A straight line scratch was made on cell layers using a sterile $1 \mathrm{ml}$ disposable serological pipette. Images of cell migration were taken using microscope at $0 \mathrm{~h}$ and $24 \mathrm{~h}$ after the scratch. The cells were counted manually at $24 \mathrm{~h}$ post scratch from 6 images and the data are presented with standard deviations.

\subsection{Transwell assay}

$48 \mathrm{~h}$ after transfection, cells were cultured in serum free medium treated with or without rSEMA7A for $12 \mathrm{~h}$. The cells then digested and suspended in serum free medium. The transwell experiments were performed in 24 well transwell plates. $200 \mu \mathrm{l}$ cell suspension was added in the upper chamber and the lower chamber was added with $800 \mu$ RPMI 1640 medium containing $10 \%$ FBS. Then the plates were incubated in $5 \% \mathrm{CO}_{2}$ under $37{ }^{\circ} \mathrm{C}$. $24 \mathrm{~h}$ later, cells were fixed with $4 \%$ paraformaldehyde for $30 \mathrm{~min}$ and the chambers were subjected to $0.2 \%$ Triton $\mathrm{X}-100$ for $15 \mathrm{~min}$ and $0.05 \%$ gentian violet dye for
$5 \mathrm{~min}$. The number of stained cells were counted under inverted microscope.

\subsection{Statistical analysis}

All data are expressed as mean \pm SD and statistical analysis were performed with one-way ANOVA followed by Dunnett's test. Statistical difference was accepted if $P<0.05$.

\section{Results}

\subsection{SEMA7A and its receptor plexin $\mathrm{C} 1$ upregulated in NSCLC A549 cells}

As shown in Fig. 1, expression of SEMA7A and its receptor plexin C1 were significantly higher in NSCLC A549 cells compared with normal human pulmonary alveolar epithelial cells both in mRNA and protein level $(P<0.001)$. However, expression of $\beta 1$-integrin in A549 cells was on levels of normal cells.

\subsection{SEMA7A and its receptor plexin C1 upregulated in NSCLC H1299 cells}

As shown in Fig. 2, expression of SEMA7A and its receptor plexin C1 were significantly higher in NSCLC H1299 cells compared with normal human pulmonary alveolar epithelial cells both in mRNA and protein level $(P<0.01)$. However, expression of $\beta 1$-integrin in H1299 cells was on levels of normal cells.
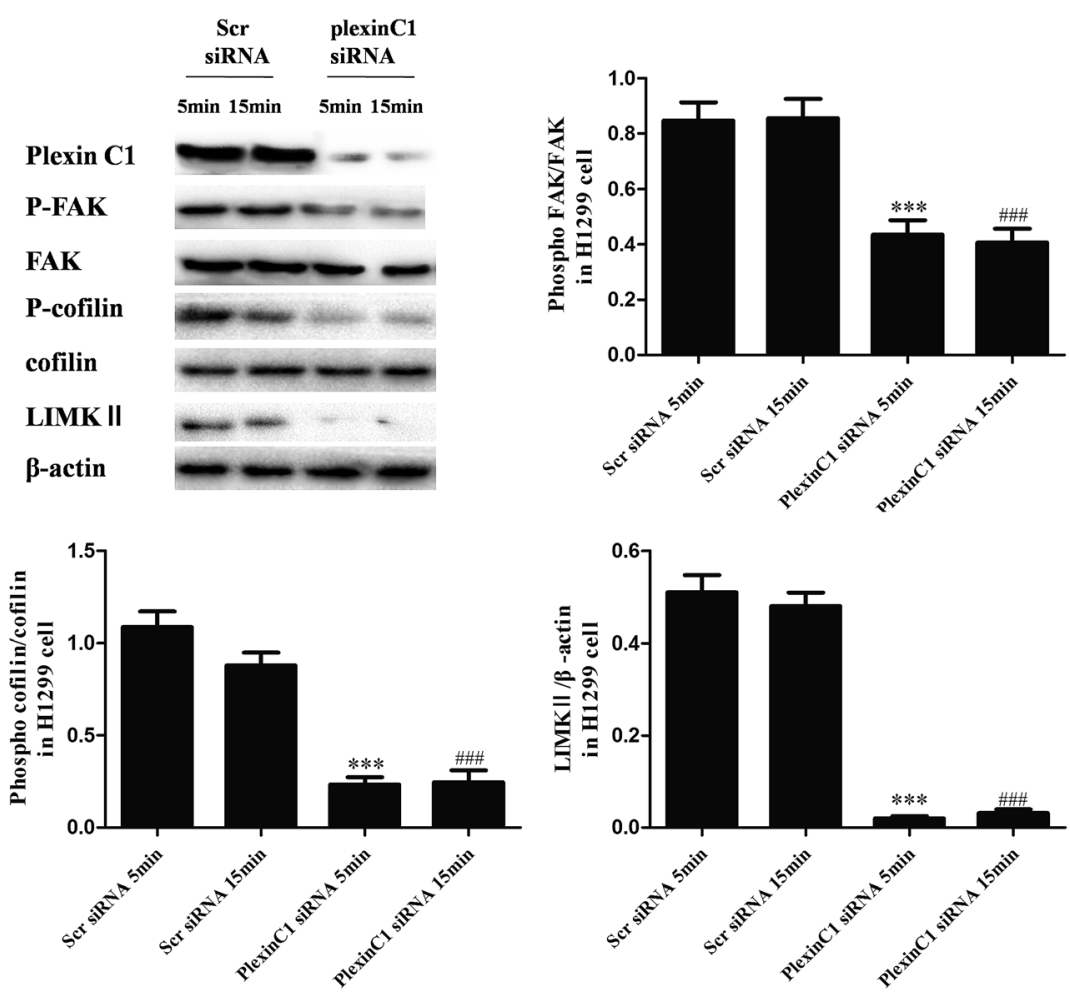

Fig. 6 Knockdown of plexin C1 decreases the expression of LIMKII, inhibits phosphorylation of FAK and cofilin in NSCLC H1299 cells. All data were obtained with $100 \mathrm{ng} \mathrm{ml}^{-1}$ rSEMA7A stimulation for 5 or 15 min. $n=8$, ***P $<0.001$ versus rSEMA7A for 5 min + scramble siRNA group, \#\#\#P $<0.001$ versus rSEMA7A for 15 min + scramble siRNA group. 


\subsection{SEMA7A activates the expression of LIMKII, stimulates} phosphorylation of FAK and cofilin in NSCLC A549 cells

To assess the role of SEMA7A on tumor migration related signaling pathways, we explored the effect of SEMA7A on activation of FAK and cofilin pathways in A549 cells using rSEMA7A. A549 cells were treated with rSEMA7A at doses ranging from 1 to $100 \mathrm{ng} \mathrm{m}{ }^{-1}$ for $15 \mathrm{~min}$. Control group was treated with vehicle. As shown in Fig. 3, the expression of LIMKII, phosphorylated FAK and cofilin were significantly promoted as the increase of rSEMA7A doses. Maximal FAK and cofilin phosphorylation were observed at $100 \mathrm{ng} \mathrm{ml}^{-1}$ and maximal LIMKII were observed at $50 \mathrm{ng} \mathrm{m} \mathrm{m}^{-1}$.

\subsection{SEMA7A activates the expression of LIMKII, stimulates phosphorylation of FAK and cofilin in NSCLC H1299 cells}

We also explored the role of SEMA7A on tumor migration related signaling pathways in $\mathrm{H} 1299$ cells. H1299 cells were treated with rSEMA7A at doses ranging from 1 to $100 \mathrm{ng} \mathrm{ml}^{-1}$ for $15 \mathrm{~min}$. Control group was treated with vehicle. As shown in Fig. 4, the expression of LIMKII, phosphorylated FAK and cofilin were significantly promoted as the increase of rSEMA7A doses.
3.5 Knockdown of plexin C1 decreases the expression of LIMKII, inhibits phosphorylation of FAK and cofilin in NSCLC A549 cells

To assess the role of plexin $\mathrm{C} 1$ on tumor migration related signaling pathways, we explored the effect of interaction between SEMA7A and plexin C1 on activation of FAK and cofilin pathways in A549 cells using rSEMA7A. 48 h after siRNA transfection, A549 cells were treated with rSEMA7A $\left(0 \mathrm{ng} \mathrm{ml}^{-1}\right.$ or 100 $\mathrm{ng} \mathrm{ml} \mathrm{m}^{-1}$ ) for 5 or $15 \mathrm{~min}$. As shown in Fig. 5A and B, knockdown of plexin $\mathrm{C} 1$ decreased the expression of LIMKII, inhibited phosphorylation of FAK and cofilin in A549 cells compared with scramble siRNA group. In addition, the effect of inhibition in 15 min group was significant compared with 5 min group.

\subsection{Knockdown of plexin $\mathrm{C} 1$ decreases the expression of LIMKII, inhibits phosphorylation of FAK and cofilin in NSCLC H1299 cells}

We also explored the role of plexin $\mathrm{C} 1$ on tumor migration related signaling pathways in H1299 cells. $48 \mathrm{~h}$ after siRNA transfection, cells were treated with rSEMA7A $\left(100 \mathrm{ng} \mathrm{ml}^{-1}\right)$ for 5 or 15 min. As shown in Fig. 6, knockdown of plexin C1 decreased the expression of LIMKII, inhibited phosphorylation
$\mathbf{A}$

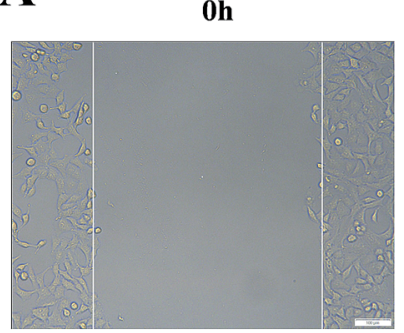

$\operatorname{rSEMA7A}(24 \mathrm{~h})$

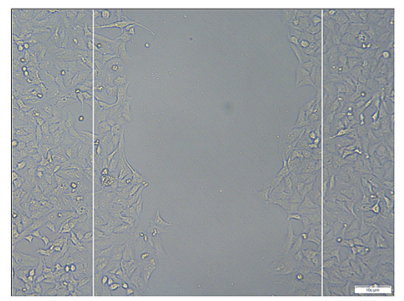

rSEMA7A+plexinC1 siRNA (24h)

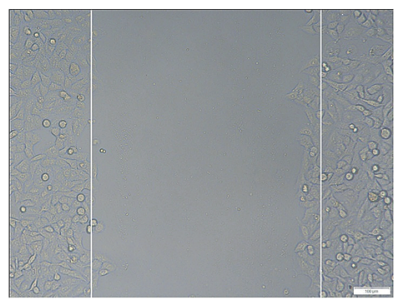

24h

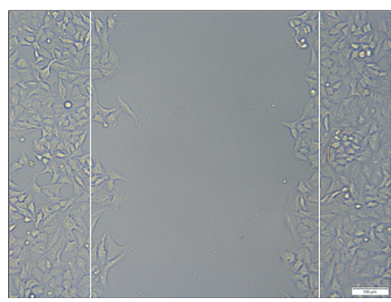

rSEMA7A+Ser SiRNA ( 24h )
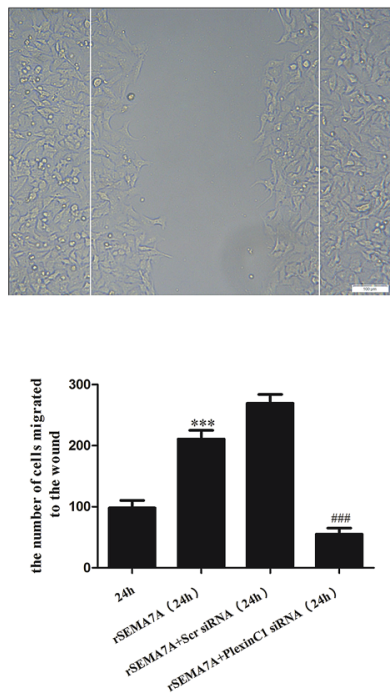

B

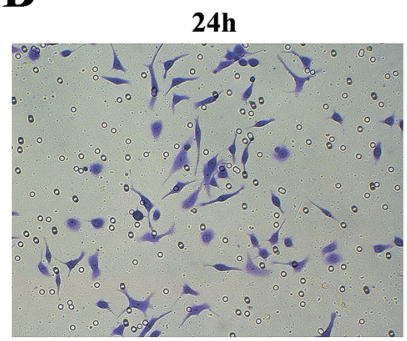

rSEMA7A+Scr siRNA

$$
\text { (24h) }
$$

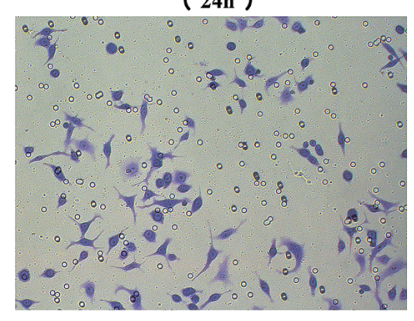
$(24 \mathrm{~h})$
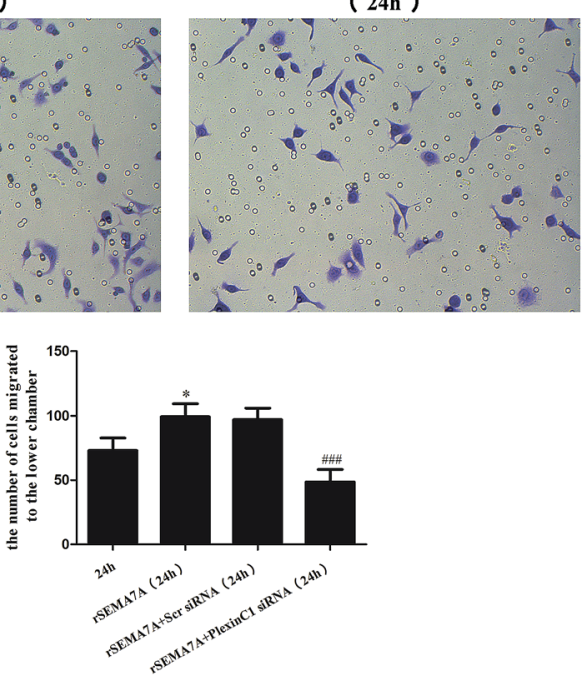

Fig. 7 Knockdown of plexin C1 inhibits the cell migration in NSCLC A549 cells stimulated by rSEMA7A. (A) Wound scratch assay of A549 cells was examined by microscope (magnification bar $=100 \mu \mathrm{m})$. (B) Transwell assay of A549 cells was examined by microscope $(200 \times) . n=6, * P<0.05$, ***P $<0.001$ versus group $24 \mathrm{~h}, \# \# \# P<0.001$ versus group rSEMA7A $24 \mathrm{~h}$. 
of FAK and cofilin in H1299 cells compared with scramble siRNA group.

\subsection{Knockdown of plexin $\mathrm{C} 1$ inhibits the cell migration in NSCLC A549 cells stimulated by rSEMA7A}

To investigate the role of plexin $\mathrm{C} 1$ on cell migration, we explored the effect of interaction between SEMA7A and plexin C1 on A549 cells using wound scratch assay and transwell assay. $48 \mathrm{~h}$ after siRNA transfection, A549 cells were treated with rSEMA7A (100 ng $\mathrm{ml}^{-1}$ ). As shown in Fig. 7A, rSEMA7A treatment promoted cell migration after $24 \mathrm{~h}$. However, treatment with plexin C1 siRNA decreased the cell migration after $24 \mathrm{~h}$. The same result is also shown in transwell assay (Fig. 7B).

\subsection{Knockdown of plexin $\mathrm{C} 1$ inhibits the cell migration in NSCLC H1299 cells stimulated by rSEMA7A}

We also investigated the role of plexin C1 on H1299 cell migration. $48 \mathrm{~h}$ after siRNA transfection, H1299 cells were treated with rSEMA7A (100 $\mathrm{ng} \mathrm{m}^{-1}$ ). As shown in Fig. 8A, rSEMA7A treatment promoted cell migration after $24 \mathrm{~h}$. However, treatment with plexin C1 siRNA decreased the cell migration after $24 \mathrm{~h}$. The same result is also shown in transwell assay (Fig. 8B).

A

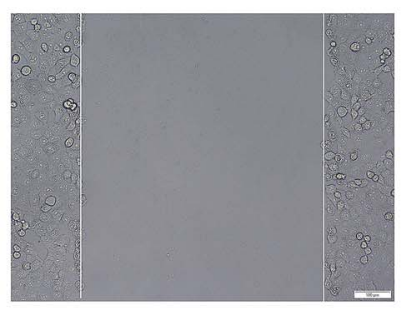

rSEMA7A ( 24h )

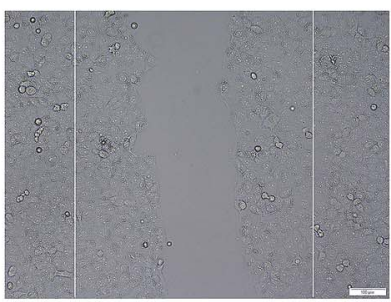

rSEMA7A+plexinC1 siRNA ( 24h)

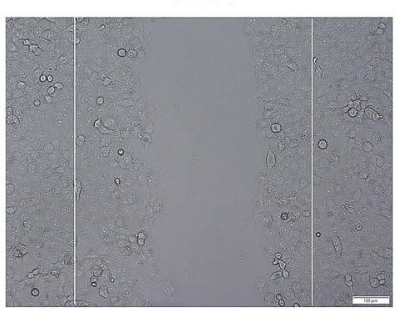

24h

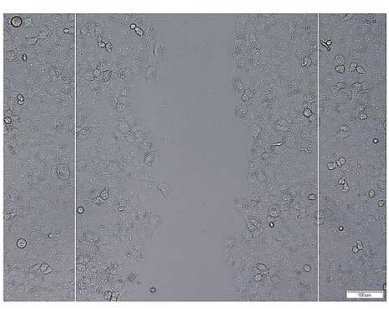

rSEMA7A+Scr SIRNA

( 24h)
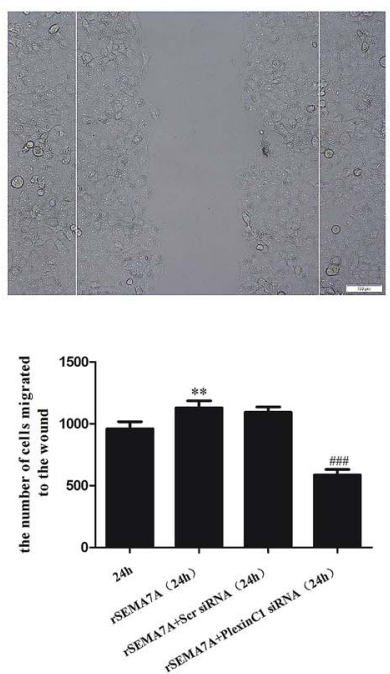

\section{Discussion}

In this study, we explored the role of SEMA7A and its receptor plexin C1 in the migration of NSCLC cells. The results showed that SEMA7A and its receptor plexin C1 were highly expressed in NSCLC A549 cells and H1299 cells. In addition, treatment with rSEMA7A activated the tumor migration related pathway FAK and cofilin in both cells. However, knockdown of plexin C1 reduced the actin-dependent cell migration, decreased the expression of LIMKII and inhibited phosphorylation of FAK and cofilin in A549 cells and H1299 cells.

SEMA7A was initially found to regulate neurite growth and axon track formation during embryonic development. ${ }^{21}$ SEMA7A is also involved in immune responses such as modulation of $\mathrm{T}$ cell function, stimulation of macrophage recruitment and pro-inflammatory cytokine production, regulation of chemokine expression and dendritic cell migration. ${ }^{\mathbf{4 - 8 , 2 2 , 2 3}}$ During the recent past years, several studies have shown that SEMA7A has effect in cancer progression. ${ }^{10-12}$ SEMA7A worked through interaction with its receptor plexin $\mathrm{C} 1$ and $\beta 1$-integrin. Previous studies have shown that $\beta 1$-integrin is associated with immunomodulatory function and plexin $\mathrm{C} 1$ was associated with cell adhesion, dendricity and cytoskeletal reorganization. ${ }^{4-7}$ In our present study, we found that SEMA7A and its receptor

\section{B}

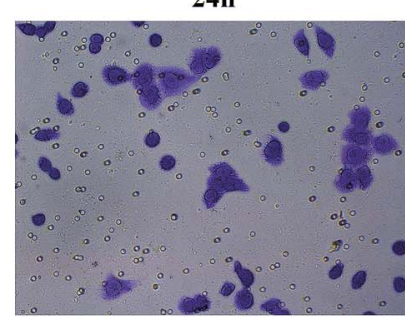

rSEMA7A+Scr SiRNA (24h)

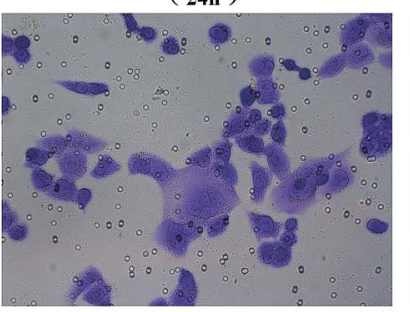

rSEMA7A+plexinC1 siRNA


Fig. 8 Knockdown of plexin C1 inhibits the cell migration in NSCLC H1299 cells stimulated by rSEMA7A. (A) Wound scratch assay of H1299 cells was examined by microscope (magnification bar $=100 \mu \mathrm{m}$ ). (B) Transwell assay of H1299 cells was examined by microscope $(200 \times) . n=6, * * P<$ 0.01 versus group $24 \mathrm{~h}$, \#\#\#P $<0.001$ versus group rSEMA7A $24 \mathrm{~h}$. 
plexin C1 were highly expressed in NSCLC A549 cells and H1299 cells. Therefore, we explored the effect of interaction between SEMA7A and plexin C1 on NSCLC A549 cell and H1299 cell migration and its related signaling pathways.

FAK and cofilin pathway are strongly linked to the tumor adhesion, migration and progression. FAK is the key component of the focal adhesion complex, which links focal adhesions to the cytosolic signaling pathway. ${ }^{24}$ Phosphorylation of FAK can regulate the cell cytoskeleton. Cofilin is an actin binding protein involved in actin disassembly and is downstream target of LIMK. ${ }^{17}$ Previous studies showed that activation of integrin induced phosphorylation of FAK. ${ }^{\mathbf{1 4}}$ However, expression of $\beta 1$ integrin in A549 cells and H1299 cells remained very low. In our study, SEMA7A activates the expression of LIMKII, stimulates phosphorylation of FAK and cofilin in A549 cells and H1299 cells. Activation of these signaling pathways ultimately induced increase of stress fibers, filopodia and membrane ruffles, promoted cell motility. Moreover, knockdown of plexin C1 inhibited FAK and cofilin phosphorylation after rSEMA7A stimulation. Most interestingly, our findings in NSCLC cell line are different with melanoma cell line in some aspects. Plexin C1 is absent in melanoma cell line. However, cofilin and its related signaling pathway are also activated in melanoma. This phenomenon is different from our findings. Unlike melanoma, plexin C1 is highly expressed in NSCLC cells and activation of cofilin is depended on interaction between SEMA7A and plexin C1. Some studies ${ }^{25}$ about melanoma indicated that plexin C1 contains an intrinsic Ras-GTPase activating domain which resulting in exchange of GTP for GDP on R-Ras. Therefore, the expression of lexin $\mathrm{C} 1$ inactivated the R-Ras. But, R-Ras is a key factor in regulation of melanoma migration. For this reason, loss of plexin $\mathrm{C} 1$ in melanoma cell line increased cell adhesion and migration via activation of R-Ras. From these difference we can see that the mechanisms of plexin $\mathrm{C} 1$ function in cell migration are multifactorial. In future studies, we will exam some other aspects between plexin $\mathrm{C} 1$ and NSCLC cell migration to make this mechanism more explicit. In addition, the mechanism of LIMKII upregulation (gene expression or post translation) is not clear in this study. We will explore the way of LIMKII upregulation in the future studies.

In summary, our experiments demonstrated the roles of SEMA7A and its receptor plexin C1 in NSCLC cell migration. These findings showed that SEMA7A and plexin C1 triggered cell migration via activating FAK and cofilin signaling pathways. It may be considered as a potential therapeutic target for the treatment of NSCLC.

\section{Competing interests}

The authors declare that there are no competing interests.

\section{Acknowledgements}

This work was supported by National Nature Science Foundation of China (No. 81570067).

\section{References}

1 A. W. Puschel, R. H. Adams and H. Betz, Murine semaphorin $\mathrm{D} /$ collapsin is a member of a diverse gene family and creates domains inhibitory for axonal extension, Neuron, 1995, 14(5), 941-948.

2 A. L. Kolodkin, D. J. Matthes and C. S. Goodman, The semaphorin genes encode a family of transmembrane and secreted growth cone guidance molecules, Cell, 1993, 75(7), 1389-1399.

3 Y. Luo, et al., A family of molecules related to collapsin in the embryonic chick nervous system, Neuron, 1995, 14(6), 11311140.

4 J. M. Roth, et al., Semaphorin 7A Aggravates Pulmonary Inflammation during Lung Injury, PLoS One, 2016, 11(1), e0146930.

5 K. Suzuki, et al., Semaphorin 7A initiates T-cell-mediated inflammatory responses through alpha1beta1 integrin, Nature, 2007, 446(7136), 680-684.

6 N. Mizutani, T. Nabe and S. Yoshino, Semaphorin 7A plays a critical role in IgE-mediated airway inflammation in mice, Eur. J. Pharmacol., 2015, 764, 149-156.

7 K. Suzuki, A. Kumanogoh and H. Kikutani, Semaphorins and their receptors in immune cell interactions, Nat. Immunol., 2008, 9(1), 17-23.

$8 \mathrm{~S}$. Holmes, et al., Sema7A is a potent monocyte stimulator, Scand. J. Immunol., 2002, 56(3), 270-275.

$9 \mathrm{~K}$. Konig, et al., The plexin $\mathrm{C} 1$ receptor promotes acute inflammation, Eur. J. Immunol., 2014, 44(9), 2648-2658.

10 R. Lazova, et al., The semaphorin 7A receptor plexin C1 is lost during melanoma metastasis, Am. J. Dermatopathol., 2009, 31(2), 177-181.

11 C. A. Formolo, et al., Secretome signature of invasive glioblastoma multiforme, J. Proteome Res., 2011, 10(7), 3149-3159.

12 M. Allegra, et al., Semaphorin-7a reverses the ERF-induced inhibition of EMT in Ras-dependent mouse mammary epithelial cells, Mol. Biol. Cell, 2012, 23(19), 3873-3881.

13 G. A. Scott, et al., Plexin C1, a receptor for semaphorin 7a, inactivates cofilin and is a potential tumor suppressor for melanoma progression, J. Invest. Dermatol., 2009, 129(4), 954-963.

14 J. Alanko and J. Ivaska, Endosomes: Emerging Platforms for Integrin-Mediated FAK Signalling, Trends Cell Biol., 2016, 26(6), 391-398.

15 R. Kanteti, et al., FAK and paxillin, two potential targets in pancreatic cancer, OncoTargets Ther., 2016, 7(21), 3158631601.

16 S. Beraud-Dufour, et al., Focal Adhesion Kinase-Dependent Role of the Soluble Form of Neurotensin Receptor-3/ Sortilin in Colorectal Cancer Cell Dissociation, Int. J. Mol. Sci., 2016, 17(11), 1860.

17 E. F. Spence and S. H. Soderling, Actin Out: Regulation of the Synaptic Cytoskeleton, J. Biol. Chem., 2015, 290(48), 2861328622. 
18 G. Kanellos and M. C. Frame, Cellular functions of the ADF/ cofilin family at a glance, $J$. Cell Sci., 2016, 129(17), 32113218.

19 S. Shishkin, et al., Cofilin-1 and Other ADF/Cofilin Superfamily Members in Human Malignant Cells, Int. J. Mol. Sci., 2016, 18(1), 10.

20 R. W. Scott and M. F. Olson, LIM kinases: function, regulation and association with human disease, J. Mol. Med., 2007, 85(6), 555-568.

21 R. J. Pasterkamp, et al., Semaphorin 7A promotes axon outgrowth through integrins and MAPKs, Nature, 2003, 424(6947), 398-405.
22 Y. Gan, et al., Role of semaphorin 7a signaling in transforming growth factor beta1-induced lung fibrosis and scleroderma-related interstitial lung disease, Arthritis Rheum., 2011, 63(8), 2484-2494.

23 A. K. Czopik, et al., Semaphorin 7A is a negative regulator of $\mathrm{T}$ cell responses, Immunity, 2006, 24(5), 591-600.

24 Q. Jiang, et al., rLj-RGD3 induces apoptosis via the mitochondrial-dependent pathway and inhibits adhesion, migration and invasion of human HeyA8 cells via FAK pathway, Int. J. Biol. Macromol., 2017, 96, 652-668.

25 Y. Chen, et al., The neural guidance receptor plexin C1 delays melanoma progression, Oncogene, 2013, 32(41), 4941-4949. 